\title{
Impact of pollution load of water and sediment of Hokersar wetland on nutrient concentration and biochemical parameters of Trapa natans L.: An economically important plant species of Kashmir, Jammu and Kashmir, India
}

\author{
Haleema Bano*, Shayesta Malik*, M. Ashraf Bhat** and Nageena Nazir*** \\ Division of Environmental Sciences, Sher-e-Kashmir University of Agricultural Sciences and Technology of Kashmir, Shalimar-190025, \\ Srinagar, Jammu and Kashmir, India \\ **Division of Genetics and Plant Breeding, Sher-e-Kashmir University of Agricultural Sciences and Technology of Kashmir, Shalimar-190 \\ 025, Srinagar, Jammu and Kashmir, India \\ ***Division of Agriculture Statistics, Sher-e-Kashmir University of Agricultural Sciences and Technology of Kashmir, Shalimar-190 025, \\ Srinagar, Jammu and Kashmir, India
}

\section{Article Info}

Article history

Received 1 May 2021

Revised 17 June 2021

Accepted 18 June 2021

Published online 30 June 2021

\section{Keywords}

Hokersar wetland

Trapa natans L.

Phosphorus

Calcium

Carotenoid

\begin{abstract}
Hokersar wetland is very important for the survival of migratory birds, but during the last four decades, the topography of the wetland has drastically changed. The wetland has reduced from 1875.04 ha in 1969 to 1300 ha in 2008 due to encroachments, sediment load carried by Doodah Ganga River and extension of willow plantations. It has shrunk and depleted due to the human settlements, domestic/ sewage pollution, pollution due to the addition of fertilizers, siltation due to floods, conversion into agricultural land, encroachments and unwanted growth of aquatic weeds, etc. Keeping these facts in view, present study titled "Impact of polluted water and sediment of Hokersar wetland on nutrient concentration and biochemical parameters of Trapa natans L.: An economically important plant species of Kashmir, Jammu and Kashmir, India". In T. natans highest mean NPK content $\left(4.07 \times 10^{4}\right.$ $\mathrm{mg} \mathrm{kg}-1,0.35 \times 10^{4} \mathrm{mg} \mathrm{kg}^{-1}$ and $\left.4.47 \times 10^{4} \mathrm{mg} \mathrm{kg}^{-1}\right)$ was recorded during summer season and lowest $(2.85$ $\times 10^{4} \mathrm{mg} \mathrm{kg}^{-1}, 0.18 \times 10^{4} \mathrm{mg} \mathrm{kg}^{-1}$ and $2.41 \times 10^{4} \mathrm{mg} \mathrm{kg}^{-1}$ ) was recorded during autumn season. With respect to sites, highest mean NPK content was recorded at Inlet site $\left(4.17 \times 10^{4} \mathrm{mg} \mathrm{kg}^{-1}, 0.30 \times 10^{4} \mathrm{mg} \mathrm{kg}^{-1}\right.$, $3.99 \times 10^{4} \mathrm{mg} \mathrm{kg}^{-1}$ ) and least at Outlet site (control site). Similar was the case with heavy metal concentration, i.e., higher concentration was recorded during summer season and lowest was recorded during autumn season. With respect to sites, highest mean heavy metal content was recorded at Inlet site and least at Outlet site (control site). The high metal concentration beyond permissible limits may damage the normal metabolism of plant. Chlorophyll ' $\mathrm{a}$ ' varied from 1.95 to $2.14 \mathrm{mg} / \mathrm{kg}$ during all three seasons. The chlorophyll ' $b$ ' content also varied from 0.253 to $0.310 \mathrm{mg} / \mathrm{kg}$ and total chlorophyll content ranged from 2.18 to $2.46 \mathrm{mg} / \mathrm{kg}$ in three different seasons. The maximum chlorophyll ' $a$ ', ' $b$ ', and total chlorophyll contents were recorded near Trapa abundance site and the minimum values were recorded at Inlet. The concentration of carotenoid varied significantly from 1.10 to $1.29 \mathrm{mg} / \mathrm{kg}$ during the entire study period. The significant increase in the above studied parameters may be attributed to high nutrient availability in the wetland because of high pollution load.
\end{abstract}

\section{Introduction}

Hokersar has been listed under National Wetlands Conservation Programme. Hokersar wetland is differentiated into three varied zones, marshy and exposed area extending from North-to-North West, Central deeper area, South Eastern side covering most of the silted area. The North-Eastern zone comprises of diverse and dense macrophytic set up and maximum numbers of macrophyte species. The central deep area is largely a free expanse of water except at certain places where two predominant species of macrophyes Trapa natans and Phragmites australis occupy a large area. The marshy

\section{Corresponding author: Dr. Haleema Bano}

Assistant Professor, Division of Environmental Sciences, Sher-eKashmir University of Agricultural Sciences and Technology of Kashmir, Shalimar-190025, Srinagar, Jammu and Kashmir, India E-mail: haleemaashraf@gmail.com Tel.: +91-7006854354 zone of the wetland and the open waters provide an efficient habitat to the migratory birds. Study by Khan et al. (2004) on the macrophyte community in relation to environmental stresses of Hokersar wetland reserves probably is the only long-term study carried out so far. The study indicates a shift in macrophyte community structure as evidenced by complete disappearance of Nelumbo nucifera and near disappearance of Eurayle ferox and Acorus calamus.

Hokersar wetland is very important for the survival of migratory birds, but during the last four decades, the topography of the wetland has drastically changed. The wetland has reduced from 1875.04 ha in 1969 to 1300 ha in 2008 due to encroachments by farmers, sediment load carried by Doodh Ganga River and extension of willow plantations. These changes in composition and structure of wetland have affected its normal functioning and deteriorated its water and sediment quality (Romshoo and Rashid, 2012). 
The wetland inhibits a number of major macrophytes like Phragmites communis (common reed), Typha latifolia (broad leaf cattail), Nelumbium nucifera (Indian lotus), Trapa natans L. (water chestnut), Ceratophyllum demersum (horn wort), etc., and minor macrophytes like Salvinia natans (floating fern), Azolla pinnata (water velvet), Wollfia polyrhiza Schield (duck weed), Lemna gibba L. (swollen duck weed), etc. (Afshan et al., 2014).

T. natans is among economically important aquatic plants. It grows as a rooted floating plant and is commonly called as Singhara. It belongs to family Lythraceae in the order Myrtales and genus Trapa. Its floating leaves are arranged in a rosette. The upper leaves are usually $2-4 \mathrm{~cm}$ long and the lower leaves reach up to $15 \mathrm{~cm}$. The petioles of the floating leaves are 0.6-1.8 $\mathrm{m}$ long. The flowers are white in color and consist of petals and 4 green sepals. The fruits are four horned nut like structure about $3 \mathrm{~cm}$ long that develops under water. Fruits ripen in about a month and can remain viable for about 12 years. Each seed can give rise to 10-15 rosettes and each rosette may produce up to 20 seeds (Bailey, 1949). In India, water chestnut is distributed all over the country and is locally introduced in to paddy fields as alternative crop (Babu and Dwivedi, 2012). Keeping all these facts in view, the present study entitled "Impact of polluted water and sediment of Hokersar wetland on chemical and biochemical parameters of $T$. natans: An economically important plant species of Kashmir, Jammu and Kashmir, India" was under taken.

\section{Materials and Methods}

\subsection{Study area}

The present research work was conducted from July 2017 to June 2018 at Sher-e-Kashmir University of Agricultural Sciences and Technology of Kashmir, Shalimar, Srinagar (J \& K). The macrophyte (Trapa natans L.) samples were collected from four different sampling sites, viz., Inlet (polluted site), Outlet (Control site), Centre and Trapa abundance site of Hokersar wetland during spring, summer and autumn seasons of the year. The samples were oven dried at $80^{\circ} \mathrm{C}$ to constant weight after washing with clean tap water. The dried samples were crushed and passed through $2 \mathrm{~mm}$ sieve and stored at room temperature for analysis.

\subsection{Methodology}

\subsubsection{Chemical parameters of $T$. natans}

Total nitrogen content in the T. natans was estimated by microKjeldahl method (Jackson, 1973). For total phosphorus, known weight of plant sample was digested in di-acid (mixture of $\mathrm{HNO}_{3}$ : $\mathrm{HClO}_{4}$ ) in the ratio of 2:1 as per procedure described by Jackson (1973). Total potassium in plant samples was determined by the method of Toth et al. (1948). For finding out mineral nutrients or heavy metals, the plant samples were digested in a di-acid mixture consisting of $\mathrm{HNO}_{3}$ and $\mathrm{HClO}_{4}$. To the known amount of plant material $(1 \mathrm{~g}), 5 \mathrm{ml}$ of concentrated $\mathrm{HNO}_{3}$ was added and kept overnight. Next day, $12 \mathrm{ml}$ of di-acid mixture $\left(\mathrm{HNO}_{3}: \mathrm{HClO}_{4}, 3: 1\right)$ was added and digested on hot plate. The remaining digested material was diluted to $25 \mathrm{ml}$ with distilled water and was then analyzed for the presence of heavy metals $(\mathrm{Mn}, \mathrm{Fe}, \mathrm{Cu}$ and $\mathrm{Zn}$ ) by using atomic absorption spectrophotometer.

Photosynthetic pigment content, i.e., the contents of chlorophyll $\mathrm{a}$, chlorophyll $\mathrm{b}$, total chlorophyll and carotenoid were determined according to the method given by Hiscox and Isrealstam (1979), using dimethyl sulphoxide (DMSO). $100 \mathrm{~g}$ of fresh leaves were homogenized in $10 \mathrm{ml}$ of DMSO and after keeping it in oven for half an hour at $40-50^{\circ} \mathrm{C}$. The absorption of solution was noted at wave lengths $(\lambda)$ of $480,510,645,663 \mathrm{~nm}$ on UV spectrophotometer.

\section{Results}

3.1 Nutrient and mineral content analysis in T. natans

\subsubsection{Nitrogen}

In $T$. natans, the maximum mean nitrogen content $\left(3.831 \times 10^{4} \pm\right.$ $0.005 \mathrm{mg} / \mathrm{kg}$ ) was recorded at Inlet followed by Trapa abundance site $\left(3.321 \times 10^{4} \pm 0.015 \mathrm{mg} / \mathrm{kg}\right)$ and least $\left(2.491 \times 10^{4} \pm 0.017 \mathrm{mg} /\right.$ $\mathrm{kg}$ ) at Outlet in spring season. The maximum mean nitrogen content $\left(4.971 \times 10^{4} \pm 0.001 \mathrm{mg} / \mathrm{kg}\right)$ was observed at Inlet followed by Trapa abundance site $\left(3.881 \times 10^{4} \pm 0.001 \mathrm{mg} / \mathrm{kg}\right)$ and minimum $\left(3.681 \times 10^{4} \pm 0.001 \mathrm{mg} / \mathrm{kg}\right)$ at Outlet site in summer season. In autumn, the highest mean nitrogen content $\left(3.72 \pm 0.0011 \times 10^{4}\right.$ $\mathrm{mg} / \mathrm{kg}$ ) was recorded at Inlet followed by Trapa abundance site $\left(2.73 \pm 0.0021 \times 10^{4} \mathrm{mg} / \mathrm{kg}\right)$ and least $\left(2.331 \times 10^{4} \pm 0.001 \mathrm{mg} / \mathrm{kg}\right)$ at Outlet. The site-wise highest mean nitrogen content $\left(4.171 \times 10^{4}\right.$ $\mathrm{mg} / \mathrm{kg})$ was recorded at Inlet and lowest $\left(2.831 \times 10^{4} \mathrm{mg} / \mathrm{kg}\right)$ at Outlet (Figure 2). However, with respect to seasons, highest mean nitrogen content $\left(4.071 \times 10^{4} \mathrm{mg} / \mathrm{kg}\right)$ was recorded during summer followed by spring $\left(3.081 \times 10^{4} \mathrm{mg} / \mathrm{kg}\right)$ and least $\left(2.81 \times 10^{4} \mathrm{mg} / \mathrm{kg}\right)$ during autumn season (Figure 1). Nitrogen content of sites and seasons was statistically significant from each other (Table 1).

\subsubsection{Phosphorus}

In T. natans, the maximum mean phosphorus content $\left(0.271 \times 10^{4}\right.$ $\pm 0.001 \mathrm{mg} / \mathrm{kg}$ ) was recorded at Inlet followed by Trapa abundance site $\left(0.231 \times 10^{4} \pm 0.009 \mathrm{mg} / \mathrm{kg}\right)$ and least $\left(0.191 \times 10^{4} \pm 0.002 \mathrm{mg} /\right.$ $\mathrm{kg}$ ) at Outlet during spring season. Maximum mean phosphorus content $\left(0.421 \times 10^{4} \pm 0.008 \mathrm{mg} / \mathrm{kg}\right)$ was observed at Inlet followed by Trapa abundance site $\left(0.371 \times 10^{4} \pm 0.003 \mathrm{mg} / \mathrm{kg}\right)$ and minimum $\left(0.281 \times 10^{4} \pm 0.005 \mathrm{mg} / \mathrm{kg}\right)$ at Outlet in summer season. In autumn, the highest mean phosphorus content $\left(0.221 \times 10^{4} \pm 0.013 \mathrm{mg} / \mathrm{kg}\right)$ was recorded at Inlet followed by Trapa abundance site $(0.241 \times$ $\left.10^{4} \pm 0.013 \mathrm{mg} / \mathrm{kg}\right)$ and least $\left(0.121 \times 10^{4} \pm 0.015 \mathrm{mg} / \mathrm{kg}\right)$ at Outlet. However, with respect to seasons, highest mean phosphorus content $\left(0.351 \times 10^{4} \mathrm{mg} / \mathrm{kg}\right)$ was recorded during summer followed by spring $\left(0.221 \times 10^{4} \mathrm{mg} / \mathrm{kg}\right)$ and least $\left(0.191 \times 10^{4} \mathrm{mg} / \mathrm{kg}\right)$ during autumn season (Figures 1,2). Phosphorus recorded at all sites and during all seasons was seen statistically significant from each other and the interaction between sites $\times$ seasons was also found significant (Table 1).

\subsubsection{Potassium}

The maximum mean potassium content $\left(3.561 \times 10^{4} \pm 0.005 \mathrm{mg} / \mathrm{kg}\right)$ was recorded at Inlet followed by Trapa abundance site $\left(3.281 \times 10^{4}\right.$ $\pm 0.008 \mathrm{mg} / \mathrm{kg})$ and least $\left(2.071 \times 10^{4} \pm 0.017 \mathrm{mg} / \mathrm{kg}\right)$ at Outlet during spring season. Maximum mean potassium content $\left(5.181 \times 10^{4} \pm\right.$ $0.001 \mathrm{mg} / \mathrm{kg}$ ) was observed at Inlet followed by Trapa abundance site $\left(4.481 \times 10^{4} \pm 0.009 \mathrm{mg} / \mathrm{kg}\right)$ and minimum $\left(3.891 \times 10^{4} \pm 0.001 \mathrm{mg} / \mathrm{kg}\right)$ at Outlet during summer. In autumn, the highest mean potassium content $\left(3.221 \times 10^{4} \pm 0.003 \mathrm{mg} / \mathrm{kg}\right)$ was recorded at Inlet followed by Trapa abundance site $\left(2.451 \times 10^{4} \pm 0.010 \mathrm{mg} / \mathrm{kg}\right)$ and least $\left(1.851 \times 10^{4} \pm\right.$ $0.003 \mathrm{mg} / \mathrm{kg}$ ) at Outlet. The site-wise highest mean potassium content in T. natans $\left(3.991 \times 10^{4} \mathrm{mg} / \mathrm{kg}\right)$ was recorded at Inlet and lowest $\left(2.601 \times 10^{4} \mathrm{mg} / \mathrm{kg}\right)$ at Outlet. However season-wise highest mean of potassium content $\left(4.471 \times 10^{4} \mathrm{mg} / \mathrm{kg}\right)$ was recorded during summer followed by spring $\left(2.881 \times 10^{4} \mathrm{mg} / \mathrm{kg}\right)$ and least $\left(2.411 \times 10^{4} \mathrm{mg} / \mathrm{kg}\right)$ in autumn season (Figures 1,2). All sites and seasons were statistically significant from each other with respect to potassium content and their interaction was also found significant (Table 1). 
Table 1: Average seasonal variation in nitrogen, phosphorus and potassium contents of $T$. natans at different sites in Hokersar wetland

\begin{tabular}{|c|c|c|c|c|c|c|c|}
\hline \multirow[b]{2}{*}{ Parameters } & \multirow[b]{2}{*}{ Seasons } & \multicolumn{4}{|c|}{ Sites } & \multirow[b]{2}{*}{ Mean } & \multirow[b]{2}{*}{ C.D $(p \leq 0.05)$} \\
\hline & & Inlet & Centre & $\begin{array}{l}\text { Trapa } \\
\text { abundance } \\
\text { site } \\
\end{array}$ & Outlet & & \\
\hline \multirow{4}{*}{$\begin{array}{l}\text { Nitrogen } \\
\left(1 \times 10^{4} \mathrm{mg} / \mathrm{kg}\right)\end{array}$} & Spring & $3.83 \pm 0.005$ & $2.68 \pm 0.015$ & $3.32 \pm 0.015$ & $2.49 \pm 0.017$ & 3.08 & \multirow{4}{*}{$\begin{array}{l}\text { Seasons }(\mathrm{S}): 0.025 \\
\text { Sites }(\mathrm{S}): 0.029 \\
\text { Seasons } \times \\
\text { Sites:0.050 }\end{array}$} \\
\hline & Summer & $4.97 \pm 0.001$ & $3.77 \pm 0.005$ & $3.88 \pm 0.001$ & $3.68 \pm 0.001$ & 4.07 & \\
\hline & Autumn & $3.72 \pm 0.001$ & $2.63 \pm 0.001$ & $2.73 \pm 0.002$ & $2.33 \pm 0.001$ & 2.85 & \\
\hline & Mean & 4.17 & 3.02 & 3.31 & 2.83 & & \\
\hline \multirow{4}{*}{$\begin{array}{l}\text { Phosphorus } \\
\left(1 \times 10^{4} \mathrm{mg} / \mathrm{kg}\right)\end{array}$} & Spring & $0.27 \pm 0.001$ & $0.21 \pm 0.004$ & $0.23 \pm 0.009$ & $0.19 \pm 0.002$ & 0.22 & \multirow{4}{*}{$\begin{array}{l}\text { Seasons }(\mathrm{S}): 0.014 \\
\text { Sites }(\mathrm{S}): 0.016 \\
\text { Seasons } \times \\
\text { Sites:0.027 } \\
\end{array}$} \\
\hline & Summer & $0.42 \pm 0.008$ & $0.34 \pm 0.002$ & $0.37 \pm 0.003$ & $0.28 \pm 0.005$ & 0.35 & \\
\hline & Autumn & $0.22 \pm 0.013$ & $0.17 \pm 0.013$ & $0.24 \pm 0.015$ & $0.12 \pm 0.015$ & 0.18 & \\
\hline & Mean & 0.30 & 0.24 & 0.28 & 0.19 & & \\
\hline \multirow{4}{*}{$\begin{array}{l}\text { Potassium } \\
\left(1 \times 10^{4} \mathrm{mg} / \mathrm{kg}\right)\end{array}$} & Spring & $3.56 \pm 0.005$ & $2.63 \pm 0.015$ & $3.28 \pm 0.008$ & $2.07 \pm 0.017$ & 2.88 & \multirow{4}{*}{$\begin{array}{l}\text { Seasons }(\mathrm{S}): 0.073 \\
\text { Sites }(\mathrm{S}): 0.084 \\
\text { Seasons } \times \\
\text { Sites:0.146 }\end{array}$} \\
\hline & Summer & $5.18 \pm 0.001$ & $4.34 \pm 0.029$ & $4.48 \pm 0.009$ & $3.89 \pm 0.001$ & 4.47 & \\
\hline & Autumn & $3.22 \pm 0.003$ & $2.12 \pm 0.020$ & $2.45 \pm 0.010$ & $1.85 \pm 0.003$ & 2.41 & \\
\hline & Mean & 3.99 & 3.03 & 3.40 & 2.60 & & \\
\hline
\end{tabular}

C.D. = Critical difference

\subsubsection{Iron}

The maximum mean iron content $(105.31 \pm 0.014 \mathrm{mg} / \mathrm{kg})$ was recorded at Inlet followed by Trapa abundance site ( $89.528 \pm 0.015$ $\mathrm{mg} / \mathrm{kg})$ and least $(76.74 \pm 0.015 \mathrm{mg} / \mathrm{kg})$ was seen at Outlet during spring season (Figures 1, 2). The maximum mean iron content $(119.96 \pm 0.012 \mathrm{mg} / \mathrm{kg})$ in $T$. natans was observed at Inlet followed by Trapa abundance site $(96.66 \pm 0.065 \mathrm{mg} / \mathrm{kg})$ and minimum $(72.85$ $\pm 0.023 \mathrm{mg} / \mathrm{kg}$ ) at Outlet site during summer. In autumn, the highest mean iron content $(79.58 \pm 0.001 \mathrm{mg} / \mathrm{kg})$ was recorded at Inlet followed by Trapa abundance site $(58.77 \pm 0.001 \mathrm{mg} / \mathrm{kg})$ and least $(46.73 \pm 0.007 \mathrm{mg} / \mathrm{kg})$ at Outlet. The site-wise highest mean iron content in $T$. natans $(101.61 \mathrm{mg} / \mathrm{kg})$ was recorded at Inlet and lowest $(65.44 \mathrm{mg} / \mathrm{kg})$ at Outlet. However, with respect to seasons, highest mean iron content $(95.42 \mathrm{mg} / \mathrm{kg})$ was recorded during summer followed by spring $(89.76 \mathrm{mg} / \mathrm{kg})$ and least $(60.38 \mathrm{mg} / \mathrm{kg})$ during autumn season. All sites and seasons were statistically significant from each other with respect to iron content and interaction between sites $\times$ seasons was also found significant (Table 2).

\subsubsection{Copper}

The maximum mean copper content $(9.81 \pm 0.025 \mathrm{mg} / \mathrm{kg})$ was recorded at Inlet followed by Trapa abundance site (8.14 \pm 0.043 $\mathrm{mg} / \mathrm{kg})$ and least $(6.91 \pm 0.059 \mathrm{mg} / \mathrm{kg})$ at Outlet in spring season (Table 33). The highest mean copper content $(16.87 \pm 0.001 \mathrm{mg} /$ $\mathrm{kg}$ ) was observed at Inlet followed by Trapa abundance site ( 9.97 $\pm 0.045 \mathrm{mg} / \mathrm{kg})$ and minimum $(7.79 \pm 0.001 \mathrm{mg} / \mathrm{kg})$ at Outlet during summer season. In autumn, the highest mean copper content (7.89 $\pm 0.032 \mathrm{mg} / \mathrm{kg}$ ) was recorded at Inlet followed by Trapa abundance site $(6.98 \pm 0.029 \mathrm{mg} / \mathrm{kg})$ and least $(5.77 \pm 0.041 \mathrm{mg} / \mathrm{kg})$ was observed at Outlet. The site-wise highest mean copper content $(11.55 \mathrm{mg} / \mathrm{kg})$ was recorded at Inlet and lowest $(6.82 \mathrm{mg} / \mathrm{kg})$ at Outlet. However, with respect to seasons, highest mean copper content $(10.88 \mathrm{mg} / \mathrm{kg})$ was recorded during summer followed by spring $(7.96 \mathrm{mg} / \mathrm{kg})$ and least $(6.75 \mathrm{mg} / \mathrm{kg})$ was recorded during autumn season (Figures 3,4). The mean copper content of sites and seasons was found statistically significant and interaction between sites $\times$ seasons was also found significant (Table 2).

\subsubsection{Manganese}

The maximum mean manganese content $(157.93 \pm 0.077 \mathrm{mg} / \mathrm{kg})$ was recorded at Inlet followed by Trapa abundance site (123.73 \pm 0.020 $\mathrm{mg} / \mathrm{kg})$ and least $(90.13 \pm 0.015 \mathrm{mg} / \mathrm{kg})$ at Outlet during spring season. Maximum mean manganese content $(235.88 \pm 0.013 \mathrm{mg} / \mathrm{kg})$ was observed at Inlet followed by Trapa abundance site (167.88 \pm 0.018 $\mathrm{mg} / \mathrm{kg})$ at and minimum $(112.84 \pm 0.019 \mathrm{mg} / \mathrm{kg})$ was recorded at Outlet in summer season. In autumn, the highest mean manganese content $(98.66 \pm 0.025 \mathrm{mg} / \mathrm{kg})$ was recorded at Inlet followed by Trapa abundance site $(75.74 \pm 0.026 \mathrm{mg} / \mathrm{kg})$ and least $(37.850 \pm 0.024 \mathrm{mg} / \mathrm{kg})$ at Outlet. The site-wise highest mean manganese content in T. natans $(164.15 \mathrm{mg} / \mathrm{kg})$ was recorded at Inlet and lowest $(80.27 \mathrm{mg} / \mathrm{kg})$ at Outlet. However, with respect to seasons, highest mean manganese content $(168.86 \mathrm{mg} / \mathrm{kg}$ ) was recorded during summer followed by spring $(122.84 \mathrm{mg} / \mathrm{kg}$ ) and least $(70.48 \mathrm{mg} / \mathrm{kg}$ ) during autumn season (Figures 3 , 4). The mean manganese of all sites and seasons was statistically significant from each other. Interaction between sites $\times$ seasons was also found significant (Table 2).

\subsubsection{Zinc}

The maximum mean zinc content $(53.87 \pm 0.015 \mathrm{mg} / \mathrm{kg})$ was recorded at Inlet followed by Trapa abundance site (43.67 \pm 0.011 $\mathrm{mg} / \mathrm{kg})$ and least $(31.73 \pm 0.015 \mathrm{mg} / \mathrm{kg})$ at Outlet during spring season (Figures 3, 4). The maximum mean zinc content (83.55 \pm $0.002 \mathrm{mg} / \mathrm{kg}$ ) in T. natans was observed at Inlet followed by Trapa abundance site $(67.94 \pm 0.008 \mathrm{mg} / \mathrm{kg})$ and minimum $(43.88 \pm 0.001$ $\mathrm{mg} / \mathrm{kg}$ ) at Outlet during summer season. In autumn, the highest 
mean zinc content $(39.93 \pm 0.021 \mathrm{mg} / \mathrm{kg})$ was recorded at Inlet followed by Trapa abundance site $(33.82 \pm 0.039 \mathrm{mg} / \mathrm{kg})$ and least $(26.61 \pm 0.017 \mathrm{mg} / \mathrm{kg})$ was observed at Outlet. The site-wise highest mean zinc content in $T$. natans $(59.11 \mathrm{mg} / \mathrm{kg})$ was recorded at Inlet and lowest $(34.07 \mathrm{mg} / \mathrm{kg}$ ) at Outlet. However, season-wise highest mean zinc content $(65.76 \mathrm{mg} / \mathrm{kg})$ was recorded during summer followed by spring $(43.03 \mathrm{mg} / \mathrm{kg})$ and least $(33.31 \mathrm{mg} / \mathrm{kg})$ in autumn season. All sites and seasons were statistically significant from each other with respect to zinc content. The interaction between sites $\times$ seasons was also found significant (Table 2 ).

Table 2: Average seasonal variation in iron, copper, manganese and zinc (mg/kg) contents of T. natans at different sites in Hokersar wetland

\begin{tabular}{|c|c|c|c|c|c|c|c|}
\hline \multirow[b]{2}{*}{ Parameters } & \multirow[b]{2}{*}{ Seasons } & \multicolumn{4}{|c|}{ Sites } & \multirow[b]{2}{*}{ Mean } & \multirow[b]{2}{*}{ C.D $(p \leq 0.05)$} \\
\hline & & Inlet & Centre & $\begin{array}{l}\text { Trapa } \\
\text { abundance } \\
\text { site }\end{array}$ & Outlet & & \\
\hline \multirow[t]{4}{*}{ Iron } & Spring & $105.31 \pm 0.014$ & $87.46 \pm 0.038$ & $89.52 \pm 0.015$ & $76.74 \pm 0.015$ & 89.76 & \multirow{4}{*}{$\begin{array}{l}\text { Seasons }(\mathrm{S}): 0.192 \\
\text { Sites }(\mathrm{S}): 0.221 \\
\text { Seasons } \times \\
\text { Sites:0.384 }\end{array}$} \\
\hline & Summer & $119.96 \pm 0.012$ & $92.21 \pm 0.041$ & $96.66 \pm 0.065$ & $72.85 \pm 0.023$ & 95.42 & \\
\hline & Autumn & $79.58 \pm 0.001$ & $56.47 \pm 0.011$ & $58.77 \pm 0.0001$ & $46.73 \pm 0.0007$ & 60.38 & \\
\hline & Mean & 101.61 & 78.71 & 79.98 & 65.44 & & \\
\hline \multirow[t]{4}{*}{ Copper } & Spring & $9.81 \pm 0.025$ & $7.01 \pm 0.049$ & $8.14 \pm 0.043$ & $6.91 \pm 0.059$ & 7.96 & \multirow{4}{*}{$\begin{array}{l}\text { Seasons }(S): 0.336 \\
\text { Sites }(S): 0.338 \\
\text { Seasons } \times \\
\text { Sites:0.672 }\end{array}$} \\
\hline & Summer & $16.87 \pm 0.001$ & $8.89 \pm 0.050$ & $9.97 \pm 0.045$ & $7.79 \pm 0.001$ & 10.88 & \\
\hline & Autumn & $7.89 \pm 0.032$ & $6.37 \pm 0.029$ & $6.98 \pm 0.021$ & $5.77 \pm 0.041$ & 6.75 & \\
\hline & Mean & 11.55 & 7.42 & 8.36 & 6.82 & & \\
\hline \multirow[t]{4}{*}{ Manganese } & Spring & $157.93 \pm 0.077$ & $119.57 \pm 0.002$ & $123.73 \pm 0.020$ & $90.13 \pm 0.015$ & 122.84 & \multirow{4}{*}{$\begin{array}{l}\text { Seasons }(S): 0.324 \\
\text { Sites }(S): 0.375 \\
\text { Seasons } \times \\
\text { Sites:0.649 }\end{array}$} \\
\hline & Summer & $235.88 \pm 0.013$ & $158.84 \pm 0.023$ & $167.88 \pm 0.018$ & $112.84 \pm 0.019$ & 168.86 & \\
\hline & Autumn & $98.66 \pm 0.025$ & $69.68 \pm 0.028$ & $75.74 \pm 0.026$ & $37.850 \pm 0.024$ & 70.48 & \\
\hline & Mean & 164.15 & 116.03 & 122.45 & 80.27 & & \\
\hline \multirow[t]{4}{*}{ Zinc } & Spring & $53.87 \pm 0.015$ & $42.86 \pm 0.034$ & $43.67 \pm 0.011$ & $31.73 \pm 0.015$ & 43.03 & \multirow{4}{*}{$\begin{array}{l}\text { Seasons }(S): 0.250 \\
\text { Sites }(S): 0.288 \\
\text { Seasons } \times \\
\text { Sites:0.499 }\end{array}$} \\
\hline & Summer & $83.55 \pm 0.002$ & $67.66 \pm 0.009$ & $67.94 \pm 0.008$ & $43.88 \pm 0.001$ & 65.76 & \\
\hline & Autumn & $39.93 \pm 0.021$ & $32.89 \pm 0.014$ & $33.82 \pm 0.039$ & $26.61 \pm 0.017$ & 33.31 & \\
\hline & Mean & 59.11 & 47.81 & 48.48 & 34.07 & & \\
\hline
\end{tabular}

C.D. = Critical difference

\subsection{Analysis of biochemical parameters in T. natans}

\subsubsection{Chlorophyll ' $a$ '}

The maximum mean chlorophyll 'a' content $(2.30 \pm 0.004 \mathrm{mg} / \mathrm{kg})$ was recorded at Trapa abundance site followed by Centre (2.10 \pm $0.003 \mathrm{mg} / \mathrm{kg})$ and least $(1.90 \pm 0.009 \mathrm{mg} / \mathrm{kg})$ at Inlet during spring season. The maximum mean chlorophyll ' a' content $(2.45 \pm 0.05$ $\mathrm{mg} / \mathrm{kg}$ ) in T. natans was observed at Trapa abundance site followed by Centre $(2.30 \pm 0.004 \mathrm{mg} / \mathrm{kg})$ and minimum $(1.85 \pm 0.010 \mathrm{mg} / \mathrm{kg})$ at Inlet site during summer season. In autumn, the highest mean chlorophyll 'a' content $(2.10 \pm 0.011 \mathrm{mg} / \mathrm{kg})$ was recorded at Trapa abundance site followed by Centre $(2.05 \pm 0.003 \mathrm{mg} / \mathrm{kg})$ and least $(1.75 \pm 0.002 \mathrm{mg} / \mathrm{kg})$ at Inlet. The site-wise highest mean chlorophyll 'a' content in T. natans $(2.28 \mathrm{mg} / \mathrm{kg})$ was recorded at Trapa abundance site and lowest $(1.88 \mathrm{mg} / \mathrm{kg})$ at Inlet. However, with respect to seasons, highest mean chlorophyll 'a' content $(2.14 \mathrm{mg} / \mathrm{kg})$ was recorded during summer followed by spring $(2.08 \mathrm{mg} / \mathrm{kg})$ and least $(1.95 \mathrm{mg} / \mathrm{kg}$ ) in autumn season (Figures 5,6$)$. All sites and seasons were statistically significant with respect to chlorophyll 'a' content and interaction between sites $\times$ seasons was also found significant (Table 3).

\subsubsection{Chlorophyll ' $b$ '}

The maximum mean chlorophyll ' $b$ ' content $(0.358 \pm 0.004 \mathrm{mg} / \mathrm{kg})$ was recorded at Trapa abundance site followed by Centre $(0.297 \pm$ $0.009 \mathrm{mg} / \mathrm{kg})$ and least $(0.256 \pm 0.008 \mathrm{mg} / \mathrm{kg})$ at Inlet during spring season. The maximum mean chlorophyll 'b' content $(0.358 \pm 0.005$ $\mathrm{mg} / \mathrm{kg}$ ) in T. natans was observed at Trapa abundance site followed by Centre $(0.314 \pm 0.006 \mathrm{mg} / \mathrm{kg})$ and minimum $(0.289 \pm 0.004 \mathrm{mg} /$ $\mathrm{kg})$ at Inlet during summer. In autumn, the highest mean chlorophyll 'b' content $(0.285 \pm 0.013 \mathrm{mg} / \mathrm{kg})$ was recorded at Trapa abundance site followed by Centre $(0.268 \pm 0.001 \mathrm{mg} / \mathrm{kg}$ and least $(0.224 \pm$ $0.003 \mathrm{mg} / \mathrm{kg}$ ) at Inlet. The site-wise highest mean chlorophyll ' $\mathrm{b}$ ' content $(0.326 \mathrm{mg} / \mathrm{kg})$ was recorded at Trapa abundance site and lowest $(0.256 \mathrm{mg} / \mathrm{kg})$ at Inlet (Figures 5, 6). However, with respect to seasons, highest mean chlorophyll ' $b$ ' content $(0.310 \mathrm{mg} / \mathrm{kg})$ was recorded during summer followed by spring $(0.297 \mathrm{mg} / \mathrm{kg})$ and least $(0.253 \mathrm{mg} / \mathrm{kg}$ during autumn season. All sites and seasons were statistically significant from each other with respect to chlorophyll ' $b$ ' and the interaction between sites $\times$ seasons was also determined significant (Table 3 ). 


\subsubsection{Total chlorophyll content}

The mean total chlorophyll content was recorded maximum $(2.65$ $\pm 005 \mathrm{mg} / \mathrm{kg})$ at Trapa abundance site followed by Centre $(2.38 \pm$ $0.003 \mathrm{mg} / \mathrm{kg})$ and least content $(2.15 \pm 0.009 \mathrm{mg} / \mathrm{kg})$ at Inlet during spring season (Table 3 ). The mean highest total chlorophyll content $(2.81 \pm 0.006 \mathrm{mg} / \mathrm{kg})$ in $T$. natans was observed at Trapa abundance site followed by Centre $(2.62 \pm 0.002 \mathrm{mg} / \mathrm{kg})$ and minimum $(2.14 \pm$ $0.008 \mathrm{mg} / \mathrm{kg}$ ) was recorded at Inlet during summer season. In autumn, the highest mean total chlorophyll content $(2.38 \pm 0.004$ $\mathrm{mg} / \mathrm{kg}$ ) was recorded at Trapa abundance site followed by Centre $(2.34 \pm 0.001 \mathrm{mg} / \mathrm{kg})$ and least $(1.97 \pm 0.007 \mathrm{mg} / \mathrm{kg})$ at Inlet. The site-wise highest mean total chlorophyll content in T. natans (2.61 $\mathrm{mg} / \mathrm{kg}$ ) was recorded at Trapa abundance site and lowest $(2.14 \mathrm{mg} /$ $\mathrm{kg}$ ) at Inlet. However, with respect to seasons, highest mean total chlorophyll content $(2.46 \mathrm{mg} / \mathrm{kg})$ was recorded during summer followed by spring $(2.37 \mathrm{mg} / \mathrm{kg})$ and least $(2.18 \mathrm{mg} / \mathrm{kg})$ was recorded during autumn season (Figures 5,6). All sites and seasons were statistically significant from each other with respect to total chlorophyll content (Table 3).

Table 3: Average seasonal variation in chlorophyll 'a', chlorophyll 'b', total chlorophyll and carotenoid content (mg/kg) contents of $T$. natans at different sites in Hokersar wetland

\begin{tabular}{|c|c|c|c|c|c|c|c|}
\hline \multirow[b]{2}{*}{$\begin{array}{l}\text { Parameters } \\
(\mathrm{mg} / \mathrm{kg})\end{array}$} & \multirow[b]{2}{*}{ Seasons } & \multicolumn{4}{|c|}{ Sites } & \multirow[b]{2}{*}{ Mean } & \multirow[b]{2}{*}{ C.D $(p \leq 0.05)$} \\
\hline & & Inlet & Centre & $\begin{array}{l}\text { Trapa } \\
\text { abundance } \\
\text { site }\end{array}$ & Outlet & & \\
\hline \multirow{4}{*}{$\begin{array}{l}\text { Chloro-phyll } \\
\text { 'a' }\end{array}$} & Spring & $1.90 \pm 0.009$ & $2.10 \pm 0.003$ & $2.30 \pm 0.004$ & $2.05 \pm 0.006$ & 2.08 & \multirow{4}{*}{$\begin{array}{l}\text { Seasons(S):0.001 } \\
\text { Sites }(\mathrm{S}): 0.001 \\
\text { Seasons } \times \\
\text { Sites:0.003 }\end{array}$} \\
\hline & Summer & $1.85 \pm 0.010$ & $2.30 \pm 0.004$ & $2.45 \pm 0.005$ & $1.97 \pm 0.008$ & 2.14 & \\
\hline & Autumn & $1.75 \pm 0.002$ & $2.05 \pm 0.003$ & $2.10 \pm 0.011$ & $1.80 \pm 0.007$ & 1.95 & \\
\hline & Mean & 1.88 & 2.08 & 2.28 & 1.95 & & \\
\hline \multirow{4}{*}{$\begin{array}{l}\text { Chloro-phyll } \\
\text { 'b' }\end{array}$} & Spring & $0.256 \pm 0.008$ & $0.297 \pm 0.009$ & $0.358 \pm 0.004$ & $0.276 \pm 0.002$ & 0.297 & \multirow{4}{*}{$\begin{array}{l}\text { Seasons }(\mathrm{S}): 0.001 \\
\text { Sites }(\mathrm{S}): 0.001 \\
\text { Seasons } \times \\
\text { Sites:0.002 }\end{array}$} \\
\hline & Summer & $0.289 \pm 0.004$ & $0.314 \pm 0.006$ & $0.358 \pm 0.005$ & $0.303 \pm 0.011$ & 0.310 & \\
\hline & Autumn & $0.224 \pm 0.003$ & $0.268 \pm 0.001$ & $0.285 \pm 0.013$ & $0.238 \pm 0.012$ & 0.253 & \\
\hline & Mean & 0.256 & 0.286 & 0.326 & 0.279 & & \\
\hline \multirow{4}{*}{$\begin{array}{l}\text { Total chloro- } \\
\text { phyll }\end{array}$} & Spring & $2.15 \pm 0.009$ & $2.38 \pm 0.003$ & $2.65 \pm 0.005$ & $2.30 \pm 0.010$ & 2.37 & \multirow{4}{*}{$\begin{array}{l}\text { Seasons }(\mathrm{S}): 0.001 \\
\text { Sites }(\mathrm{S}): 0.001 \\
\text { Seasons } \times \\
\text { Sites:0.002 }\end{array}$} \\
\hline & Summer & $2.14 \pm 0.008$ & $2.62 \pm 0.002$ & $2.81 \pm 0.006$ & $2.27 \pm 0.012$ & 2.46 & \\
\hline & Autumn & $1.97 \pm 0.007$ & $2.34 \pm 0.001$ & $2.38 \pm 0.004$ & $2.04 \pm 0.011$ & 2.18 & \\
\hline & Mean & 2.14 & 2.38 & 2.61 & 2.23 & & \\
\hline \multirow{4}{*}{$\begin{array}{l}\text { Carotenoid } \\
\text { content }\end{array}$} & Spring & $0.87 \pm 0.001$ & $1.26 \pm 0.006$ & $1.45 \pm 0.003$ & $1.26 \pm 0.003$ & 1.21 & \multirow{4}{*}{$\begin{array}{l}\text { Seasons(S):N/S } \\
\text { Sites }(\mathrm{S}): 0.067 \\
\text { Seasons } \times \\
\text { Sites:0.116 }\end{array}$} \\
\hline & Summer & $0.95 \pm 0.001$ & $1.51 \pm 0.001$ & $1.60 \pm 0.048$ & $1.11 \pm 0.001$ & 1.29 & \\
\hline & Autumn & $0.75 \pm 0.083$ & $1.16 \pm 0.001$ & $1.28 \pm 0.010$ & $1.21 \pm 0.010$ & 1.10 & \\
\hline & Mean & 0.856 & 1.31 & 1.44 & 1.19 & & \\
\hline
\end{tabular}

C.D. = Critical difference

\subsubsection{Carotenoid}

The carotenoid content was recorded maximum $(1.45 \pm 0.003 \mathrm{mg} /$ $\mathrm{kg})$ at Trapa abundance site followed by Center $(1.26 \pm 0.006 \mathrm{mg} /$ $\mathrm{kg})$ at and least $(0.87 \pm 0.001 \mathrm{mg} / \mathrm{kg})$ was seen at inlet during spring season. The highest carotenoid content $(1.60 \pm 0.048 \mathrm{mg} / \mathrm{kg})$ in T. natans was observed at Trapa abundance site followed by Center $(1.51 \pm 0.001 \mathrm{mg} / \mathrm{kg})$ at and minimum $(0.95 \pm 0.001 \mathrm{mg} / \mathrm{kg})$ was recorded at inlet site during summer (Table 3 ). In autumn, the highest carotenoid content $(1.28 \pm 0.010 \mathrm{mg} / \mathrm{kg})$ was recorded at Trapa abundance site followed by Center $(1.16 \pm 0.001 \mathrm{mg} / \mathrm{kg})$ and least $(0.750 \pm 0.003 \mathrm{mg} / \mathrm{kg})$ was observed at Inlet. The site-wise highest mean carotenoid content in $T$. natans $(1.44 \mathrm{mg} / \mathrm{kg})$ was recorded at Trapa abundance site and lowest $(0.856 \mathrm{mg} / \mathrm{kg})$ at Inlet. However, with respect to season, highest mean carotenoid content $(1.29 \mathrm{mg} /$ $\mathrm{kg})$ was recorded during summer followed by spring $(1.211 \mathrm{mg} / \mathrm{kg})$ and least was recorded during autumn season $(1.10 \mathrm{mg} / \mathrm{kg}$ ) (Figures
$5,6)$. The mean carotenoid content of sites and interaction between sites $\times$ seasons was statistically significant but seasons were determined statistically non-significant (Table 3).

\section{Discussion}

Phosphorus (P) is an essential structural component of cell membranes and nucleic acids and necessary for maintaining the vital biological activities in plant. Phosphorus plays an important role in photosynthesis, respiration, energy storage, cell division, cell enlargement and several other processes in plants. Mean phosphorus content in T. natans ranged from $0.18 \times 10^{4}$ to $0.35 \times$ $10^{4} \mathrm{mg} / \mathrm{kg}$ in all three seasons. Highest mean phosphorus content in T. natans was recorded at Inlet site and lowest at Outlet site (Table 1). This may attributed to the availability of nutrient load at inlet site. The findings were in agreement with findings of Smal and Olszewska (2008) and Ruiz and Velasco (2010). The highest mean $\mathrm{P}$ content was observed during summer followed by spring and 
lowest in autumn. The decrease in $\mathrm{pH}$ due high rate of decomposition in sediments with enhanced microbial activity, could be the main reason in accumulation of more $\mathrm{P}$ in summer than autumn and spring. Phosphorus showed negative correlation with all mineral contents and biochemical parameters (Table 4). The present findings are in agreement with the findings reported by Eid et al. (2012).

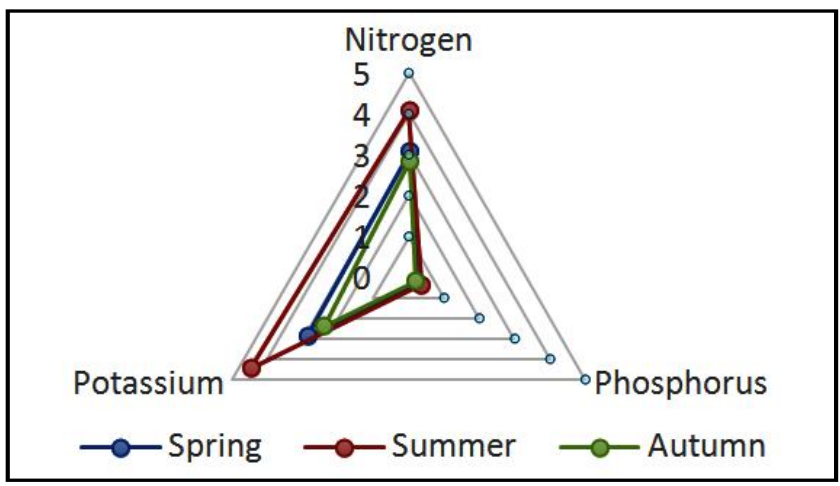

Figure 1: Seasoiial variation in $\mathrm{N}\left(1 \times 10^{4} \mathrm{mg} / \mathrm{kg}\right), \mathrm{P}\left(1 \times 10^{4} \mathrm{mg} /\right.$ $\mathrm{kg})$ and $\mathrm{K}\left(1 \times 10^{4} \mathrm{mg} / \mathrm{kg}\right)$ content of $T$. natans.

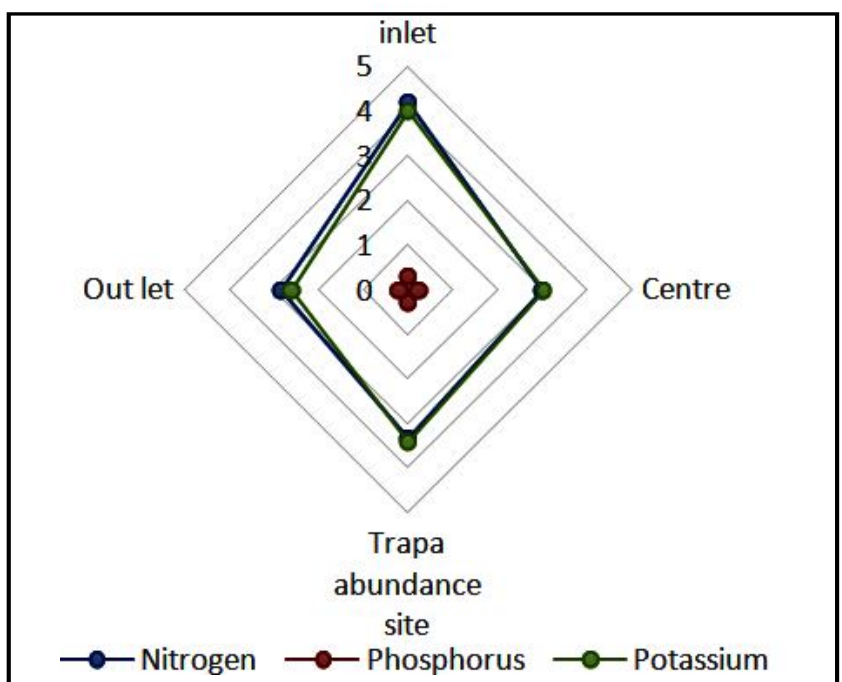

Figure 2: Variation in $\mathrm{N}\left(1 \times 10^{4} \mathrm{mg} / \mathrm{kg}\right), \mathrm{P}\left(1 \times 10^{4} \mathrm{mg} / \mathrm{kg}\right)$ and $\mathrm{K}\left(1 \times 10^{4} \mathrm{mg} / \mathrm{kg}\right)$ content of $T$. natans at different sites.

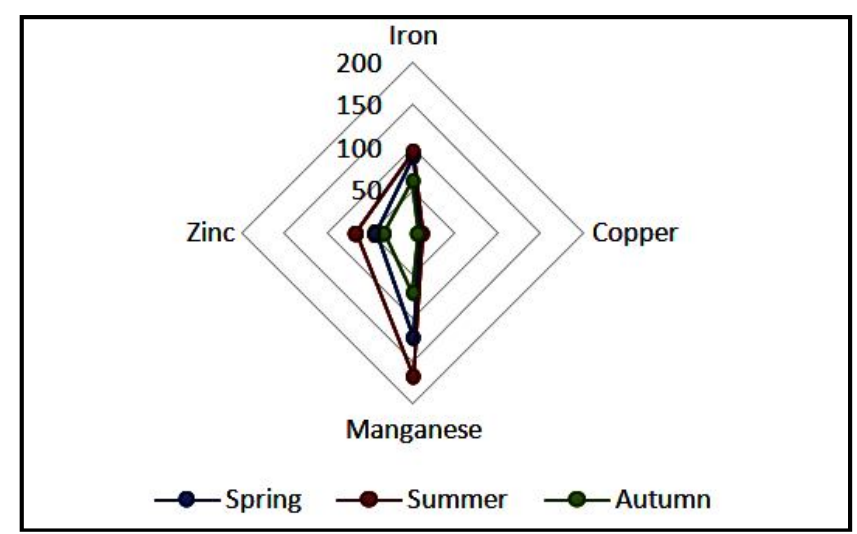

Figure 3: Seasonal variation in mineral content of $T$. natans ( $\mathrm{mg} / \mathrm{kg})$.

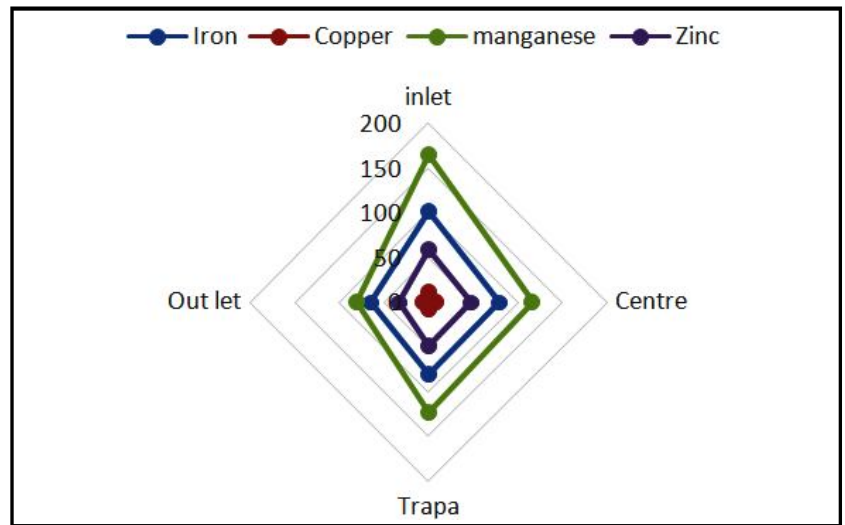

Figure 4: Variation in mineral content of $T$. natans $(\mathrm{mg} / \mathrm{kg})$ at different sites.

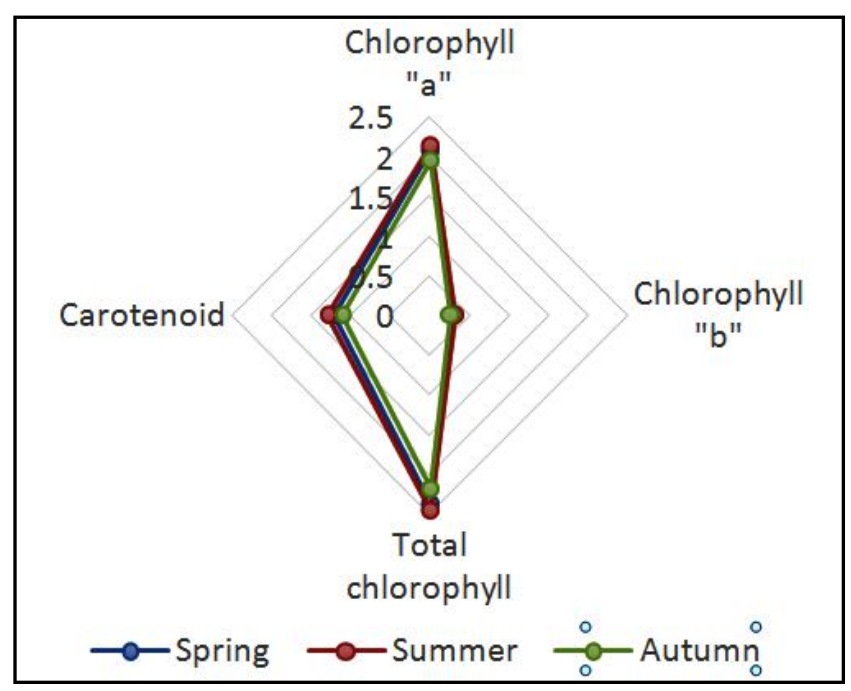

Figure 5: Seasonal variation in chlorophyll 'a', chlorophyll 'b', total chlorophyll and carotenoid content (mg/ kg) contents of $T$. natans.

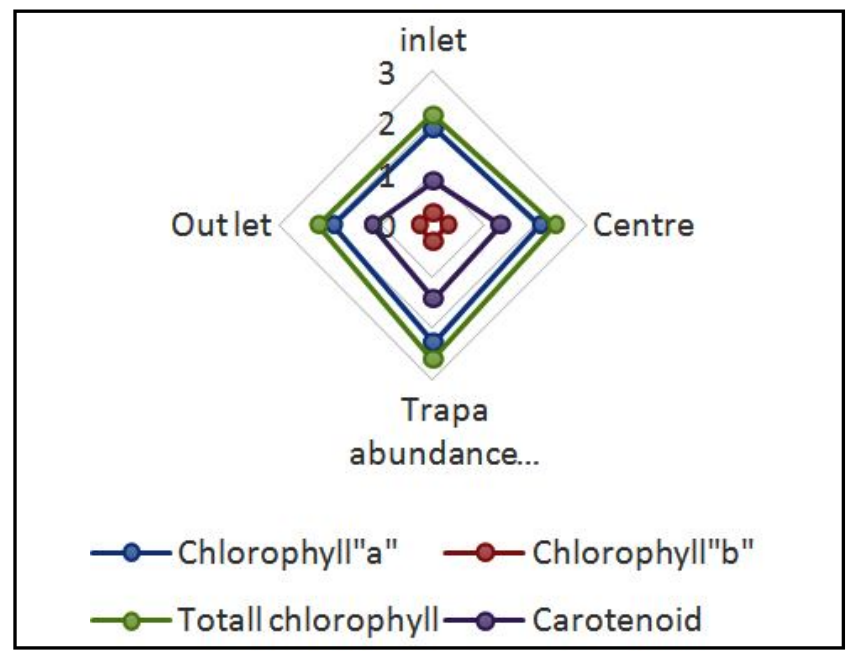

Figure 6: Variation in chlorophyll 'a', chlorophyll 'b', total chlorophyll and carotenoid content ( $\mathrm{mg} / \mathrm{kg}$ ) content of T. natans at different sites. 
Potassium (K) belongs to the group of major macronutrients, responsible for plant growth and primary production. It affects the plant shape, size, colour and taste. It regulates the opening and closing of stomata and is essential for the production of adenosine triphosphate (ATP). The mean concentration of $\mathrm{K}$ in Hokersar wetland ranged from $2.41 \times 10^{4}$ to $4.47 \times 10^{4} \mathrm{mg} / \mathrm{kg}$ during all the three seasons. High accumulation of $\mathrm{K}$ in T. natans was recorded at Inlet site and lowest at Outlet (Table 1). This may due to the high nutrient load in the form of pollution influx from sediments, agricultural fields and natural activities. These findings are in concordance with the findings reported by Vardanyan and Ingole (2006) and Parvaiz et al. (2013). Highest content of K was recorded in summer followed by spring and lowest in autumn season (Table 1). The higher mean $\mathrm{K}$ content in $T$. natans during summer may be due to the availability of $\mathrm{K}$ in sediments by way of discharge of waste water, agricultural sewage and weathering of rocks. Since, aquatic plants absorb wide range of macro and micronutrients in the polluted environment and subsequently accumulate them in their tissues. Similar findings were reported by (Parvaiz et al. (2013) and Eid et al. (2012).

Manganese (Mn) is a plant micronutrient and is naturally occurring element found in rock, soil and water. Manganese is one of nine essential nutrients that plants require for growth. Many processes are dependent on this nutrient, including chloroplast formation, photosynthesis, nitrogen metabolism and synthesis of some enzymes. Manganese content significantly varied between 70.48 to $168.86 \mathrm{mg} / \mathrm{kg}$ in T. natans. Higher mean Mn content seen at Inlet site, may be because of higher accumulations of $\mathrm{Mn}$ in sediment due to heavy pollution load. The macrophyes have a great potential to absorb trace elements along with nutrients. The content of $\mathrm{Mn}$ in exceeded the natural content $(52 \mathrm{mg} / \mathrm{kg})$ as given by Brooks and Robinson (1998), but was within the toxic range $(50-500 \mu \mathrm{g} / \mathrm{g}$ ) given by Allen (1989) and Bonanno (2013). The excessive amount of $\mathrm{Mn}$ is directly related to addition sewage sludge and the injudicious use of fertilizers in the adjoining agricultural fields. Furthermore, highest mean Mn content was seen during summer season followed by spring and lowest in autumn (Table 2). The high availability of Mn during summer season may be due to the discharge of untreated water, agricultural run-off and weathering of rocks. The results are in agreement with Fitzgerald et al. (2003), Deng et al. (2004) and Klink et al. (2013).

Copper $(\mathrm{Cu})$ is an important micronutrient for plants and is a constituent of enzymes which catalyze oxidative reactions in variety on metabolic pathways. It also activates enzymes which are involved in lignin synthesis, but at high concentration, it can be very toxic. It is an essential component in photosynthesis, plant respiration and carbohydrate metabolism. In the present study, $\mathrm{Cu}$ content ranged from 6.75 to $10.88 \mathrm{mg} / \mathrm{kg}$ in T. natans during all the three seasons. High mean concentration of $\mathrm{Cu}$ was reported at Inlet site and lower content at Outlet site (Figures 3, 4). This suggests the intense anthropogenic influence due to settlements near Inlet within the catchment area of the wetland. However, highest mean $\mathrm{Cu}$ content was seen during summer followed by spring and least in autumn (Table 2). This may be because of direct discharge or wet and dry depositions of contaminants in aquatic ecosystems. Similar findings were reported by Kumar et al. (2008).
Zinc ( $\mathrm{Zn})$ is one of the eight essential micronutrients needed by plants. It is key component of many proteins and enzymes and helps the plant to produce chlorophyll. It contributes to the hormone production and internode elongation. The mean concentration of $\mathrm{Zn}$ content in T. natans ranged from 33.31 to $65.76 \mathrm{mg} / \mathrm{kg}$ during the entire study period. Maximum mean $\mathrm{Zn}$ content was found at Inlet site as compared to Outlet (Figures 3, 4). This may be attributed to high pollution load at Inlet due to application of high amount of fertilizers in the agricultural fields and waste water discharge from households. These findings were in agreement with Fitzgerald et al. (2003), Deng et al. (2004), Baldontiniet al. (2009) and Klink et al. (2013). High mean content of $\mathrm{Zn}$ in T. natans was recorded during summer season followed by spring and lowest in autumn (Table 2). This is because of high concentration of $\mathrm{Zn}$ in sediments trigger the absorption by macrophyes as the rate of photosynthesis is higher during this period because of favorable environmental conditions, hence more Zn get accumulated in plants during summer season. Similar observations were reported by Bragato et al. (2009), Duman et al. (2007), Lessage et al. (2007) and Maddison et al. (2009). Zinc showed significant positive correlation with all mineral contents and with nitrogen and potassium parameters (Table 4).

Iron $(\mathrm{Fe})$ is plant uptake iron in its oxidized forms $\mathrm{Fe}^{2+}$ and $\mathrm{Fe}^{3+}$ forms. It is an important constituent of proteins involved in the transfer of electrons like ferredoxin and cytochrome. It activates catalase, peroxidase and nitrogenase enzyme and is essential for synthesis of chlorophyll. Iron is required in large amounts in comparison to other micronutrients. Fe content significantly varied from 60.38 to $95.42 \mathrm{mg} / / \mathrm{kg}$ during three different seasons. The highest mean Fe content in T. natans was reported at Inlet site and lowest at Outlet (Table 3). This could be attributed to the pollution load, caused by both natural as well as anthropogenic activities. The availability and accumulation of micronutrients by aquatic plants depends upon several factors like trace elements, content in bottom sediment, plants species present in water, climatic factors and related factors (Fitzgerald et al., 2003; Deng et al., 2004; Klink et al., 2013). Furthermore, with respect to seasons, highest mean Fe content $T$. natans was reported during summer followed by spring and lowest during autumn (Table 2). The high availability of Fe could be due to the discharge of domestic sewage, use of fertilizers, dumping of hospital wastes and decomposition of organic matter due to high rate of microbial activity with increased temperature in sediments readily absorbs by roots of water plants and eventually accumulates in their tissues. The present results are in concordance with results reported by Parzych et al. (2015). According to Allen (1989), 40 - $500 \mathrm{mg} \mathrm{g}^{-1} \mathrm{Fe}$ concentrations are considered as toxic to plants. The results indicated higher concentration of Fe during different seasons as well as at different sites also. Heavy metals in higher concentration may damage the normal metabolism plants.

\subsection{Biochemical analysis of T. natans}

In the present study, chlorophyll 'a' varied from 1.95 to $2.14 \mathrm{mg}$ / $\mathrm{kg}$ during all three seasons. The chlorophyll ' $\mathrm{b}$ ' content also varied from 0.253 to $0.310 \mathrm{mg} / \mathrm{kg}$ and total chlorophyll content ranged from 2.18 to $2.46 \mathrm{mg} / \mathrm{kg}$ in three different seasons. The maximum chlorophyll 'a', 'b', and total chlorophyll contents were recorded 
near Trapa abundance site and the minimum values were recorded at Inlet. The concentration of carotenoid varied significantly from 1.10 to $1.29 \mathrm{mg} / \mathrm{kg}$ during the entire study period. The significant increase in the above mentioned parameters may be attributed to high nutrient availability at the mentioned sites, which is in agreement with Taher-u-Zaman and Kushari (2002). Furthermore, all the biochemical parameters were recorded highest during summer season followed by spring and lowest in autumn (Table 3).

Table 4: Correlation between available nutrients, mineral content and biochemical parameters of T. natans growing at different sites in Hokersar wetland

\begin{tabular}{|c|c|c|c|c|c|c|c|c|c|c|}
\hline & $\mathbf{N}$ & $\mathbf{P}$ & $\mathbf{K}$ & Mn & $\mathrm{Cu}$ & $Z_{n}$ & $\mathrm{Fe}$ & C.a & C.b & T.C \\
\hline $\mathrm{P}$ & .168 & & & & & & & & & \\
\hline $\mathrm{K}$ & $.954 * *$ & .003 & & & & & & & & \\
\hline $\mathrm{Mn}$ & $.898 * *$ & -.118 & $.917 * *$ & & & & & & & \\
\hline $\mathrm{Cu}$ & $.889 *$ & -.058 & $.831 * *$ & $.914 * *$ & & & & & & \\
\hline $\mathrm{Zn}$ & $.893 * *$ & -.121 & $.947 * *$ & $.964 * *$ & $.885 * *$ & & & & & \\
\hline $\mathrm{Fe}$ & $.826 * *$ & -.023 & $.817 * *$ & $.956 * *$ & $.829 * *$ & $.867 * *$ & & & & \\
\hline C.a & .070 & -.436 & .271 & .241 & -.013 & .291 & .214 & & & \\
\hline C.b & 0.279 & -.047 & .294 & .149 & .231 & .278 & -.021 & .170 & & \\
\hline T.C & 0.105 & -.452 & .308 & .269 & .026 & .320 & .228 & $.997 * *$ & .169 & \\
\hline $\mathrm{Ca}$ & -.448 & -.564 & -.256 & .413 & -.456 & -.315 & -.559 & .395 & .215 & .400 \\
\hline
\end{tabular}

$* p<0.05$ and $* * p<0.01$; where, C. $\mathrm{a}=$ chlorophyll "a", C. $\mathrm{b}=$ Chlorophyll "b", T.C = Total Chlorophyll, Ca = Carotenoids

\section{Conclusion}

Presence of higher concentration of NPK and heavy metal content at Inlet site in the macrophyte is indication of the fact that Inlet site is highly polluted as compared to Outlet site (control site). High heavy metal concentration in the macrophyte recorded during summer season and lowest during autumn season may be because of high degradation rate of wastes during summer season. The biochemical parameters in T. natans, viz., chlorophyll 'a' $(2.28 \mathrm{mg} /$ $\mathrm{kg})$, chlorophyll 'b' $(0.326 \mathrm{mg} / \mathrm{kg})$, total chlorophyll $(2.61 \mathrm{mg} / \mathrm{kg})$ and carotenoid $(1.44 \mathrm{mg} / \mathrm{kg})$ were seen maximum at Trapa abundance site and lowest $\{(1.88 \mathrm{mg} / \mathrm{kg}, 0.256 \mathrm{mg} / \mathrm{kg}, 2.14 \mathrm{mg} / \mathrm{kg}$ and 0.856 $\mathrm{mg} / \mathrm{kg}$ ) $\}$ at Inlet site. Thus, it can be said that $T$. natans have good potential to absorb nutrients and accumulate trace elements from polluted environment.

\section{Acknowledgements}

This work was supported in the form of lab facility by Division of Environmental Sciences and Division of Soil Sciences of SKUASTKashmir, India 190025.

\section{Conflict of interest}

The authors declare that there are no conflicts of interest relevant to this article.

\section{References}

Afshan, A.; Mahajan, D. M. and Saptarshi, P.G. (2014). Macrophytes diversity in Hokersar wetland: A ramsar site (Kashmir Himalaya). National Conference: $10^{\text {th }}$ and $11^{\text {th }}$.

Allen, S.E. (1989), Chemical Analysis of Ecological Material, 2nd ed., pp:368, Blackwell Sci., Oxford, U.K.
Babu, M. and Devidi, D. H. (2012). Evaluation of biochemical attributes of water chestnut (Trapa natans var. bispinosa Roxb.) collected from Lucknow region. The Asian Journal of Horticulture, 7(2):442444.

Bonanno, G. (2013), Comparative performance of trace element bioaccumulation and biomonitoring in the plant species typha domingensis, Phragmites australis and Arun dodonax, Ecotoxicol. Environ. Safe, 97:124-130.

Bonnano, G. (2011). Trace element accumulation and distribution in the organs of Phragmites australis (common reed) and biomonitoring applications. Ecotoxic Environment Safe, 74:1057-1064.

Bragato, C.; Schiavon, M.; Polese, R.; Ertani, A.; Pittarello, M. and Malagoli, M. (2009). Seasonal variation of $\mathrm{Cu}, \mathrm{Zn}, \mathrm{Ni}$ and $\mathrm{Cr}$ concentration in phragmites baustralis Trin. Ex Steudel in a constructed wetland of North Italy. Desalination, 246:35-44.

Brooks, R.R. and B.H. Robinson (1998), Aquatic phytoremediation by accumulator plants, in plants that hyper accumulate heavy metals: Their role in phytoremediation, microbiology, archaeology, mineral exploration and phytomining, edited by R.R. Brooks, pp:203-226, CAB International, Oxon.

Deng, H.; Ye, Z. H. and Wong M. H. (2004). Accumulation of lead, zinc, copper and cadmium by 12 wetland plant species thriving in metalcontaminated sites in China, Environment Pollution, 132:29-40.

Duman, F.; Cicek, M. and Sezen, G. (2007). Seasonal changes of metal accumulation and distribution in common club rush (Schoenoplectus lacustris) and common reed (Phragmites australis), Ecotoxicology, 16:457-463.

Eid, E. M.; Shaltout, K.H.; Al-Sodany, Y.M.; Soetaert, K. and Jensen, K. (2010). Modeling growth, carbon allocation and nutrient budget of Phragmites australis in Lake Burullus, Egypt. Wtlands, 30:240251.

Eid, E. M.; Shaltout; K. H.; El-Shiekh, M.A. and Asaeda, T. (2012). Seasonal courses of nutrients and heavy metals in water, sediment and abive and below-ground Typha domingensis biomass in Lake Burullus (Egypt): Perspective for phytoremediation Flora, 207:783794. 
Fitzgerald, E. J.; Caffrey, J. M.; Nesaratnam, S. T. and Mc-Loughlin, P. (2003) Copper and lead concentrations in salt marsh plants on the Suir estuary, Ireland. Environmental Pollution, 123:67-74.

Khan, M. A.; Shah, M. A.; Mir, S. S. and Bashir, S. (2004). The environmental status of an aquatic plant communities and eco-restoration measures. Lakes and Reservoirs. Research and Management, 9(2): $125-132$.

Khan, M. A.; Shah, M.A.; Mir, S. S. and Suzana, B. (2004). The environmental status of a Kashmir Himalayan wetland game reserve: Aquatic plant communities and eco-restoration measures. Lakes and Reservoires: Research and Management, (Blackwell, Australia), 9(2):125-132.

Klink, A.; Wislocka, M.; Musial, M. and Krawczyk, J. (2013). Macro-and traceelements accumulation in Typha angustifolia L. and Typha latifolia L. organs and their use in bioindycation. Poland Journal of Environmental Studies, 22(1):183-190.

Lesage, E.; Rousseau, D. P. L.; Meers, E.; Tack, F. M. G. and De-pauw, N. (2007). Accumulation of metals in a horizontal subsurface flow constructed wetland treating domestic waste water in Flanders, Belgium, Science Total Environment, 380:102-115.

Maddison, M.; Soosaar, K.; Mauring, T. and Mander, U. (2009). The biomass and nutrient and heavy metal content of cattails and reeds in wastewater treatment wetlands for the production of construction material in Estonia. Desalinatin, 246:120-128.
Parvaiz, A.L.; Ajay, K.B. and Fayaz, A.B. (2013). A study of comparative purification efficiencies of two species of Potomegeton (Submerged macrophte) in waste water treatment. International Journal of Scientific and Research Publications, 3(1):1-4.

Parzych, A.E.; Cymer, M.; Jonczak, J. and Szymczyk, S. (2015). The ability of leaves and rhizomes of aquatic plants to accumulate macro and micronutrients. Journal of Ecological Engineering, 16(3):198-205.

Romshoo, S. A. and Rashid, I. (2012). Assessing the impacts of changing land cover and climate on Hokersar Wetland in Kashmir Himalayas. Arabian Journal of Geosciences, 7(1):143-160.

Ruiz, M. and Velasco, J. (2010). Nutrient bioaccumulation in Phragmitesaustralis: management tool for reduction of pollution in the Mar Menor. Water Air Soil Pollution, 205:173-185.

Sharma, P.; Aseada, T.; Manatunge, J. and Fujino, T. (2006). Nutrient cycling in a natural stand of Typha angustifolia. Journal of Fresh water Ecology, 21:431-438.

Smal, H. and Olszeweska, M. (2008). The effect of afforestation with Scot pines of sandy post-arable soils on selected properties. II. Reaction, carbon, nitrogen and phosphorus. Plant and Soil, 305:171-187.

Taher-u-Zaman and Khushari (2002). Evaluation of some common macrophytes cultivated in enriched water as possible source of protein and biogas. Aquatic ecology, 23:207-121.

Vardanyan, L. G. and Ingole, B. S. (2006). Studies of heavy metal accumulation in aquatic macrophytes from Sevan (America) Carambolim (India) lake systems, Environment International, 32:208-218.

Haleema Bano, Shayesta Malik, M. Ashraf Bhat and Nageena Nazir (2021). Impact of pollution load of water and sediment of Hokersar wetland on nutrient concentration and biochemical parameters of Trapa natans L.: An economically important plant species of Kashmir, Jammu and Kashmir, India. Ann. Phytomed., 10(1):298-306. http://dx.doi.org/10.21276/ap.2021.10.1.32 